\title{
Pulsation of the $\delta$ Scuti star VZ Cancri
}

\author{
J.-N. Fu ${ }^{1}$ and S.-Y. Jiang ${ }^{2}$ \\ 1 Laboratoire d'Astrophysique de Toulouse, Observatoire Midi-Pyrénées, 14 avenue Édouard Belin, F-31400 Toulouse, France \\ e-mail: fu@astro.ast.obs-mip.fr \\ 2 Beijing Astronomical Observatory, Chinese Academy of Sciences, Beijing 100080, China \\ e-mail: jianglh@public.east.cn.net
}

Received August 25, 1997; accepted January 5, 1999

\begin{abstract}
After the "O - C" technique is used to study the pulsation of the $\delta$ Scuti star VZ Cnc with the times of maximum light listed in the literature and a newlydetermined time based on new photometry for this star, some high-quality photometry light curves in the literature are analysed by the Fourier decomposition, showing that there are more than 2 frequencies in the pulsation of this star, which might be one possible explanation of the existence of the large scatter in the " $\mathrm{O}-\mathrm{C}$ " diagram.
\end{abstract}

Key words: $\delta$ Sct variable - VZ Cnc — oscillations

\section{Introduction}

The variability of VZ Cnc (HD 73857) was discovered by Whitney (1950). Since then this star has been referred to as a RR Lyrae or a Cluster-Type variable (Joy \& Wilson 1950; Guman 1955; Fitch 1955), as a supershortperiod Cepheid (Berdnikov 1975) and as dwarf Cepheid (e.g., Percy et al. 1980) or $\delta$ Scuti-type variable (Cox et al. 1984; López de Coca et al. 1990). Ferro et al. (1994) refer to VZ Cnc as a large amplitude $\delta$ Scuti star. Fitch (1955) and Cox et al. (1984) identify two periodicities in the light variations of VZ Cnc: 0.17836376 and 0.1428041 days as the first and second overtone respectively. A beat period of 0.716292 days is also indicated.

With the observed times of maximum light and the "O - C" technique, many authors have studied the period variations of VZ Cnc (Todoran 1976; Percy et al. 1980; Jiang \& Yang 1982; Cao \& Jiang 1991; Ferro et al. 1994). Four different interpretations are suggested by researchers as: a sloping linear fit, a sinusoidal fit, abrupt period changes, and long-term effects of nonlinear combinations of short frequencies. However, due to the large scatter in the $(\mathrm{O}-\mathrm{C})$ residuals, it is difficult to favor a

Send offprint requests to: J.-N. Fu case although the latter two possibilities are regarded to be unrealistic by Ferro et al. (1994).

\section{New observations and data reduction}

As a new attempt to study its possible period variation by the "O - C" technique, photometry on VZ Cnc was made at Xinglong station of Beijing Astronomical Observatory with a $60 \mathrm{~cm}$ reflector plus a CCD camera on December 19, 1995. The filter used was a Johnson $V$. After careful extinction correction on the raw magnitudes of the variable star, one new time of maximum light is determined thus: $T_{\max }=$ HJD 2450071.2820. This new time is combined with the times listed in the literature and 138 times of maximum light are collected and then listed in Table 1.

Besides this, some high-quality observation data for VZ Cnc are collected from the literature. Table 2 lists the journal of these observations.

\section{3. "O - C" calculation}

The "O - C" calculation for the collected times of maximum light of VZ Cnc was performed with the computer program OMC (Fu et al. 1998). First, a linear fit is made by the formula of $C_{1}=T_{1}+P_{1} E$. The results of fitting are: $T_{1}=$ HJD $2431550.7187, P_{1}=0.178363665$ days, and $\sigma_{1}=0.0062$ days. Then, a quadratic curve is used to fit the times as $C_{\mathrm{Q}}=T_{2}+P_{2} E+0.5 \beta E^{2}$. The fitting parameters are: $T_{2}=$ HJD 2431550.7199, $P_{2}=0.178363585$ days, $\beta=7.910^{-13}$ days/cycle, and $\sigma_{2}=0.0062$ days. The $(\mathrm{O}-\mathrm{C})_{1}$ diagram and the fit curve using the quadratic function are shown in Fig. 1.

From Fig. 1, it may be found that the "O-C" calculation does not provide reasonable explanation on the period variation of $\mathrm{VZ}$ Cnc with the existence of large scatter for the fitting, and there may be more frequencies in the pulsation of this star since large scatter is present even in one 
Table 1. Times of light maxima of VZ Cnc

\begin{tabular}{|c|c|c|c|c|c|c|c|c|c|c|c|c|c|c|}
\hline To. & $\begin{array}{c}\text { HJD } \\
2400000.0+\end{array}$ & $E$ & $\begin{array}{r}(\mathrm{O}-\mathrm{C})_{1} \\
(\text { day })\end{array}$ & ef. & No. & $\begin{array}{c}\text { HJD } \\
2400000.0+\end{array}$ & $E$ & $\begin{array}{r}(\mathrm{O}-\mathrm{C})_{1} \\
(\text { day })\end{array}$ & $\mathrm{H}$ & No. & $\begin{array}{c}\text { HJD } \\
2400000.0+\end{array}$ & $E$ & $\begin{array}{r}(\mathrm{O}-\mathrm{C})_{1} \\
(\text { day })\end{array}$ & f. \\
\hline 1 & 31550.7100 & 0.0 & -0.0087 & Wh & 47 & 34476.4195 & 16403.0 & 0.0016 & $\mathrm{Gu}$ & 93 & 42099.5045 & 59142.0 & 0.0019 & $\mathrm{Ha}$ \\
\hline 2 & 33631.8630 & 668.0 & -0.0029 & $\mathrm{Fi}$ & 48 & 34667.6237 & 17475.0 & 0.0000 & $\mathrm{Gu}$ & 94 & 42100.3915 & 9147.0 & -0.0029 & 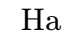 \\
\hline 3 & 33633.8220 & 1679.0 & -0.0059 & $\mathrm{Fi}$ & 49 & 34708.6487 & 17705.0 & 0.0013 & $\mathrm{Gu}$ & 95 & 42128.3910 & 04.0 & -0.0065 & 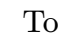 \\
\hline 4 & 33647.9205 & 758.0 & 0.0018 & $\mathrm{Fi}$ & 50 & 68 & 17875.0 & -0.0024 & $\mathrm{Fi}$ & 96 & 4213 & 93260 & 0.0080 & \\
\hline 5 & 33655.7721 & 802.0 & 0.0054 & $\mathrm{Fi}$ & 51 & 34740.9363 & 17886.0 & 0.0051 & $\mathrm{Fi}$ & 97 & 42161.3885 & 9.0 & & \\
\hline 6 & 33679.8396 & 37.0 & -0.0062 & $\mathrm{Fi}$ & 52 & 34742.8946 & 17897.0 & 0.0014 & $\mathrm{Fi}$ & 98 & 4245 & 127.0 & 060 & Го \\
\hline 7 & 33685.7355 & 1970.0 & 0.0037 & $\mathrm{Fi}$ & 53 & 34745.9325 & 17914.0 & 0.0071 & $\mathrm{Fi}$ & 99 & 42474.4160 & 1244.0 & -0.0070 & Гo \\
\hline 8 & 33686.7967 & 1976.0 & -0.0053 & $\mathrm{Fi}$ & 54 & 34746.8148 & 17919.0 & -0.0024 & $\mathrm{Fi}$ & 100 & 42476.3790 & 55.0 & -0.0060 & Гo \\
\hline 9 & 33711.7755 & 116.0 & 0.0026 & $\mathrm{Fi}$ & 55 & 34746.9902 & 17920.0 & -0 & $\mathrm{Fi}$ & 01 & 42 & & 039 & \\
\hline 10 & 6261 & 32.0 & -0.0006 & $\mathrm{Fi}$ & 56 & 3474 & 179 & 22 & $\mathrm{Fi}$ & 02 & 42 & 1.0 & 029 & \\
\hline 11 & 33722.6478 & 77.0 & -0.0052 & $\mathrm{Fi}$ & 57 & 3474 & 179 & 006 & $\mathrm{Fi}$ & 03 & 425 & 4.0 & 074 & Го \\
\hline 12 & 33730.6729 & 222.0 & -0.0065 & $\mathrm{Fi}$ & 58 & 34758 & 17987.0 & 0.0023 & $\mathrm{Fi}$ & 04 & 425 & 63.0 & 0.0075 & Го \\
\hline 13 & 33739.4205 & 2271.0 & 0.0013 & $\mathrm{Gu}$ & 59 & 34770.3620 & 18051.0 & 0.0008 & $\mathrm{Gu}$ & 105 & 42756 . & 25.0 & -0.0047 & Гo \\
\hline 14 & 33740.3175 & 76.0 & 0.0064 & $\mathrm{Gu}$ & 60 & 34770 . & 2.0 & 41 & $\mathrm{Gu}$ & 06 & 4277 & .0 & 30 & 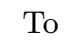 \\
\hline 15 & 750 & 2.0 & -0. & $\mathrm{Gu}$ & 61 & 34772 & .0 & 08 & $\mathrm{Gu}$ & 07 & 427 & & 88 & O \\
\hline 16 & 3374 & 93.0 & -0.0092 & $\mathrm{Gu}$ & 62 & 3477 & 2.0 & 13 & $\mathrm{Fi}$ & 88 & 428 & 9.0 & 066 & Го \\
\hline 17 & 33744.4125 & 2299.0 & -0.0009 & $\mathrm{Gu}$ & 63 & 34776.7873 & 18087.0 & 0.0050 & $\mathrm{Fi}$ & 109 & 42867 & 3447.0 & 0.0018 & Го \\
\hline 18 & 33746.3685 & 310.0 & -0.0069 & $\mathrm{Gu}$ & 64 & 34779.2770 & 18101.0 & 24 & $\mathrm{Gu}$ & 110 & 42871 & 9.0 & -0.0047 & Го \\
\hline 19 & 33758.6827 & 379.0 & 0.0002 & $\mathrm{Fi}$ & 65 & 34779. & 181 & 12 & $\mathrm{Gu}$ & 111 & 42 & .0 & -0 & Lo \\
\hline 20 & 36 & 5.0 & -0 . & $\mathrm{Fi}$ & 66 & 347 & .0 & 24 & $\mathrm{Gu}$ & 12 & 43 & & 23 & $\mathrm{Ce}$ \\
\hline 21 & 3377 & 3.0 & -0.0 & $\mathrm{Fi}$ & 67 & 347 & 18 & 60 & $\mathrm{Fi}$ & 13 & 43 & & 051 & $\mathrm{Ce}$ \\
\hline 22 & 34015.8857 & 821.0 & 0.0028 & $\mathrm{Fi}$ & 68 & $347 \varepsilon$ & 18116.0 & -0.0021 & $\mathrm{Fi}$ & 14 & 431 & 8.0 & -0.0019 & $3 \mathrm{~S}$ \\
\hline 23 & 34032.8216 & 16.0 & -0.0059 & $\mathrm{Fi}$ & 69 & 347 & 18208.0 & 0.0014 & $\mathrm{Gu}$ & 115 & 43 & & 68 & $3 S$ \\
\hline 24 & 34034.7904 & 927.0 & 0.0009 & $\mathrm{Fi}$ & 70 & 34798.5395 & 18209.0 & -0.0 & $\mathrm{Gu}$ & 16 & 43528.5 & .0 & -0 & $3 \mathrm{~S}$ \\
\hline 25 & 340 & 3.0 & 49 & $\mathrm{Fi}$ & 71 & 347 & 0.0 & -0.0007 & $\mathrm{Fi}$ & 17 & 43 & .0 & 57 & 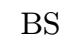 \\
\hline 26 & 3403 & 9.0 & -0.0005 & $\mathrm{Fi}$ & 72 & 3479 & 182 & 36 & $\mathrm{Fi}$ & 18 & 460 & 8.0 & 059 & $\mathrm{~J}$ \\
\hline 27 & 340 & & 0.0030 & $\mathrm{Fi}$ & 73 & & & -0 . & $\mathrm{Fi}$ & 119 & & & -0 & CJ \\
\hline 28 & 34039.7848 & 955.0 & 0.0012 & $\mathrm{Fi}$ & 74 & 35901.5400 & 24393.0 & -0.0036 & $\mathrm{PO}$ & 120 & 46095.2 & 4.0 & -0 . & CJ \\
\hline 29 & 34039.9578 & 3956.0 & -0.0042 & $\mathrm{Fi}$ & 75 & 36598.4200 & 28300.0 & 0.0096 & $\mathrm{Ah}$ & 121 & 47501.6092 & 89429.0 & 063 & $\mathrm{Pi}$ \\
\hline 30 & 340 & 9.0 & 34 & $\mathrm{Gu}$ & 76 & 36 & 8.0 & 54 & $\mathrm{Ah}$ & 122 & $47 \subseteq$ & 4.0 & 39 & $\mathrm{~J}$ \\
\hline 31 & 340 & 3.0 & 55 & $\mathrm{Fi}$ & 77 & 3660 & 28 & 12 & $\mathrm{Ah}$ & 23 & 4820 & 0.0 & 090 & CJ \\
\hline 32 & 34044 & 33.0 & 0.0028 & $\mathrm{Fi}$ & 78 & 36613 & 28384.0 & & $\mathrm{Ah}$ & 124 & 4822 & 4.0 & 067 & CJ \\
\hline 33 & 34046.7455 & 3994.0 & 0.0057 & $\mathrm{Fi}$ & 79 & 38357.9670 & 38165.0 & -0.0010 & FW & 125 & 48237.3499 & 4.0 & -0.0031 & CJ \\
\hline 34 & 34063.3487 & 1087.0 & 0.0210 & $\mathrm{Gu}$ & 80 & 38369.0310 & 38227.0 & 0.0045 & FW & 126 & 48240.2019 & 93570.0 & -0 . & CJ \\
\hline 35 & 3406 & 95.0 & 21 & $\mathrm{Fi}$ & 81 & 384 & 38 & 80 & $\mathrm{FV}$ & 127 & 48240.3776 & 1.0 & 076 & CJ \\
\hline 36 & 3406 & & & $\mathrm{Fi}$ & 82 & 391 & & 0.0030 & FW & 128 & 4902 & & 016 & $\mathrm{Fe}$ \\
\hline 37 & 34068.8593 & 4118.0 & 0.0024 & $\mathrm{Fi}$ & 83 & 39852.6396 & 46545.0 & -0.0159 & $\mathrm{Ha}$ & 129 & 49027.8720 & 97986.0 & 0.0112 & $\mathrm{Fe}$ \\
\hline 38 & 34071.7099 & 14134.0 & -0.0008 & $\mathrm{Fi}$ & 84 & 39878.5006 & 46690.0 & -0.0176 & $\mathrm{Ha}$ & 130 & 49028.7540 & 97991.0 & 0.0014 & $\mathrm{Fe}$ \\
\hline 39 & 34109.7031 & 14347.0 & 0.0009 & $\mathrm{Fi}$ & 85 & 39879.3958 & 46695.0 & -0.0142 & $\mathrm{Ha}$ & 131 & 49030.7280 & 98002.0 & 0.0134 & $\mathrm{Fe}$ \\
\hline 40 & 343 & & -0.0067 & $\mathrm{Gu}$ & 86 & 39903 & 0.0 & -0.0161 & $\mathrm{Ha}$ & 132 & 49034.6360 & 98 & -0 . & $\mathrm{Fe}$ \\
\hline 41 & 34393.4925 & 5938.0 & 0.0137 & $\mathrm{Gu}$ & 87 & 40622.3014 & 50860.0 & 0.0067 & Po & 133 & 49036.7780 & 98036.0 & -0.0010 & $\mathrm{Fe}$ \\
\hline 42 & 34393.6558 & 15939.0 & -0.0014 & $\mathrm{Gu}$ & 88 & 40676.3384 & 51163.0 & -0.0005 & Po & 134 & 49037.6690 & 98041.0 & -0.0018 & $\mathrm{Fe}$ \\
\hline 43 & 34453.3980 & 16274.0 & -0.0110 & $\mathrm{Gu}$ & 89 & 40677.4203 & 51169.0 & 0.0112 & Po & 135 & 49038.7490 & 98047.0 & 0.0080 & $\mathrm{Fe}$ \\
\hline 44 & 34455.3600 & 16285.0 & -0.0110 & $\mathrm{Gu}$ & 90 & 40686.3272 & 51219.0 & -0.0001 & Po & 136 & 49071.7415 & 98232.0 & 0.0032 & $\mathrm{Fe}$ \\
\hline 45 & 34456.4470 & 6291.0 & 0.0058 & $\mathrm{Gu}$ & 91 & 40694.3473 & 51264.0 & -0.0063 & Po & 137 & 49074.7643 & 98249.0 & -0.0061 & $\mathrm{Fe}$ \\
\hline 46 & 34457.3370 & 16296.0 & 0.0040 & $\mathrm{Gu}$ & 92 & 40890.5484 & 52364.0 & -0.0053 & PO & 138 & 50071.2820 & 103836.0 & -0.0062 & $\mathrm{Pt}$ \\
\hline
\end{tabular}

$\begin{array}{llll}\text { *Ah: } & \text { Ahnert (1959) } & \text { *Ha: } & \text { Hahn et al. (1975) } \\ \text { *BS: } & \text { Balona \& Stobie (1983) } & \text { *Pi: } & \text { Piersimoni et al. (1993) } \\ \text { *Ce: } & \text { Cester et al. (1977) } & \text { *PO: } & \text { Ponsen \& Oosterhoff (1966) } \\ \text { *CJ: } & \text { Cao \& Jiang (1991) } & \text { *Po: } & \text { Popovici (1971) } \\ \text { *Fe: } & \text { Ferro et al. (1994) } & \text { *pp: } & \text { present paper } \\ \text { *Fi: } & \text { Fitch (1955) } & \text { *To: } & \text { Todoran (1976) } \\ \text { *FW: } & \text { Fitch et al. (1966) } & \text { *Wh: } & \text { Whitney (1950). } \\ \text { *Gu: } & \text { Guman (1955) } & & \end{array}$


Table 2. Journal of the observations for VZ Cnc in the literature

\begin{tabular}{cclcccccc}
\hline Publication & Year & Site & Telescope & Filter & Nights & points & $\sigma$ & Filename \\
\hline Ferro et al. (1994) & 1993 & Loluz Obs. & $0.57 \mathrm{~m}$ & $V$ & 12 & 627 & 0.1707 & VZ1 \\
\hline \multirow{2}{*}{ Jiang et al. (1982) } & $1962-1963$ & \multirow{2}{*}{ Beijing } & \multirow{2}{*}{$0.15 \mathrm{~m}$} & $V$ & 6 & 177 & 0.1711 & VZ2a \\
& & & & $U$ & 5 & 169 & 0.2363 & VZ2b \\
& & & 20 inch & $V$ & 12 & 675 & 0.1844 & VZ2c \\
\hline Spinrad (1960) & 1959 & Leuschner \& Lick Obs. & VZ3 \\
\hline \multirow{2}{*}{ Fitch (1955) } & $1950-1951$ & Yerkes Obs. & 12 inch & $V$ & 15 & 295 & 0.2055 & VZ4a \\
& $1951-1952$ & Steward Obs. & 36 inch & $V$ & 17 & 519 & 0.1995 & VZ4b \\
& $1953-1954$ & Steward Obs. & 36 inch & $V$ & 16 & 622 & 0.2126 & VZ4c \\
\hline
\end{tabular}

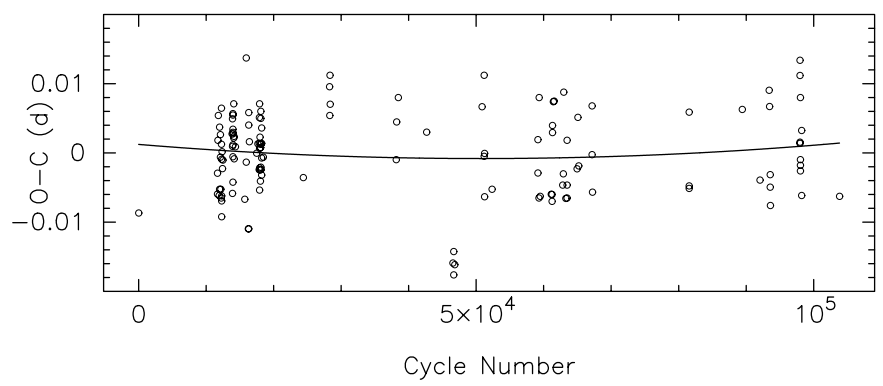

Fig. 1. The $(\mathrm{O}-\mathrm{C})$ diagram and the fit curve by a parabolic function. Large scatter is found between the observation points and the fit curve

observation season. In this case, the high-quality observation data sets listed in Table 2 are used to do multiple frequency analysis to study the pulsation of VZ Cnc.

\section{Multiple frequency determination}

\subsection{Multi-frequency analysis}

Among the data sets listed in Table 2, VZ2a, VZ2b, VZ2c have few points and VZ4a, VZ4b, VZ4c, although they include more measurements, show mainly increasing segments with few decreasing parts (see Fig. 7), which are not available to deep multiple frequency analyses due to the artificial sampling effect. So the data set VZ1 and VZ3 are used to do Fourier transformation.

The multiple-frequency analyses were performed with the computer program MFA (Hao 1991). After the nonequal spacing Fourier transformation (Deeming 1975) was done, the highest power peak was chosen automatically, or the peak was chosen by hand, then both linear and nonlinear least square fits (Hao 1991) were used to determine the best frequency and corresponding amplitude and phase. The data were then prewhitened with that frequency and the process repeated to search for the next frequency automatically. Step by step, the best frequencies and fit results were obtained. To check the reliability of the results, the theoretical light curves were calculated using the following formula,

$m(t)=m_{0}+\sum_{i} A_{i} \cos \left[2 \pi f_{i}\left(t-t_{0}\right)+\phi_{i}\right]$
Table 3. Results of the Fourier analysis for VZ1

\begin{tabular}{rrrccc}
\hline No. & \multicolumn{2}{c}{ Frequency } & Amplitude & Phase & Mark \\
& $(\mathrm{c} / \mathrm{d})$ & $(\mu \mathrm{Hz})$ & $(\mathrm{mag})$ & & \\
\hline$f_{1}$ & 5.6066 & 64.891 & 0.2003 & 0.537 & $\nu_{0}$ \\
$f_{2}$ & 11.2128 & 129.777 & 0.0748 & 0.492 & $2 \nu_{0}$ \\
$f_{3}$ & 7.0022 & 81.043 & 0.0688 & 0.996 & $\nu_{1}$ \\
$f_{4}$ & 16.8188 & 194.662 & 0.0345 & 0.444 & $3 \nu_{0}$ \\
$f_{5}$ & 12.6095 & 145.943 & 0.0302 & 0.828 & $\nu_{0}+\nu_{1}$ \\
$f_{6}$ & 1.3957 & 16.154 & 0.0250 & 0.203 & $\nu_{1}-\nu_{0}$ \\
\hline
\end{tabular}

Table 4. Results of the Fourier analysis for VZ3

\begin{tabular}{lrrccc}
\hline No. & \multicolumn{2}{c}{ Frequency } & Amplitude & Phase & Mark \\
& $(\mathrm{c} / \mathrm{d})$ & $(\mu \mathrm{Hz})$ & $(\mathrm{mag})$ & & \\
\hline$F_{1}$ & 5.6063 & 64.888 & 0.2063 & 0.423 & $\nu_{0}$ \\
$F_{2}$ & 11.2132 & 129.782 & 0.0882 & 0.242 & $2 \nu_{0}$ \\
$F_{3}$ & 7.0029 & 81.052 & 0.0637 & 0.326 & $\nu_{1}$ \\
$F_{4}$ & 16.8177 & 194.649 & 0.0475 & 0.122 & $3 \nu_{0}$ \\
$F_{5}$ & 12.6070 & 145.914 & 0.0308 & 0.125 & $\nu_{0}+\nu_{1}$ \\
$F_{6}$ & 1.3947 & 16.142 & 0.0248 & 0.676 & $\nu_{1}-\nu_{0}$ \\
\hline
\end{tabular}

where $t_{0}$ is the beginning time; $A_{i}$, the amplitude; and $\phi_{i}$, the phase. The result of the analysis provides the multiplefrequency solution and the residual, as well as various power spectra where the best multiple frequency fits have been prewhitened.

The analysis results on data set VZ1 and VZ3 are listed in Tables 3 and 4 . Figures 2 and 3 show the spectral windows and power spectra of VZ1 and VZ3, respectively. In Figs. 4 and 5, the observation points and fit light curves of VZ1 and VZ3 are shown respectively. The residuals of VZ1 and VZ3 after 6 frequency fittings are 0.021 mag and $0.027 \mathrm{mag}$, respectively.

\subsection{Fit to other data sets}

As mentioned above, the other data sets are not suitable to do independent multiple-frequency analysis. So the 6 frequencies obtained from the analysis for VZ1 
WINDOW-VZ Cnc

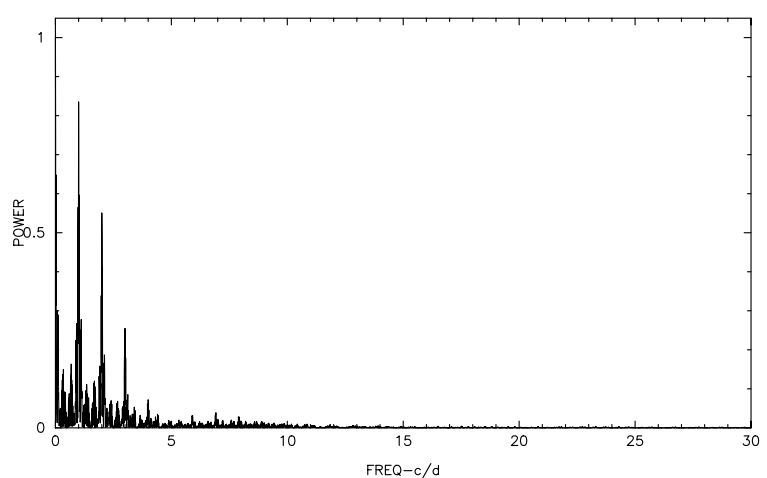

ST(02)-VZ Cnc

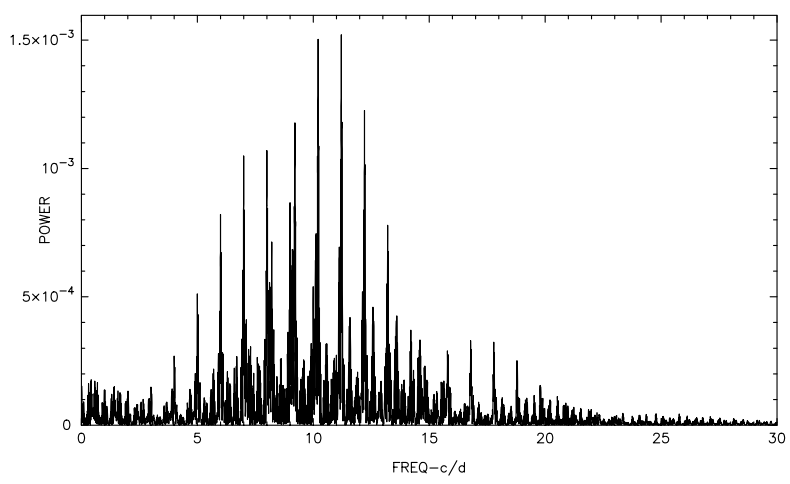

ST(04)-VZ Cnc

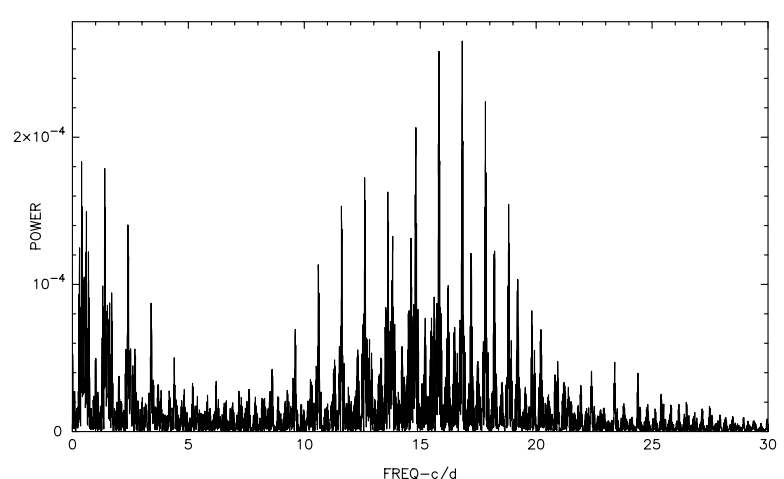

ST(06)-VZ Cnc

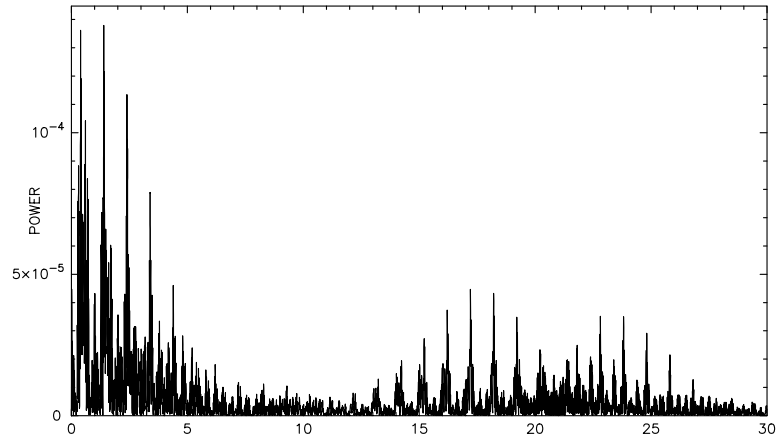

ST(01)-VZ Cnc

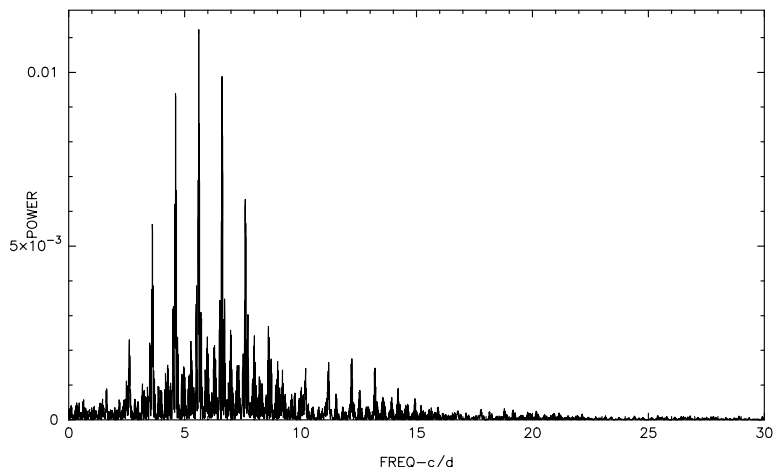

ST(03)-VZ Cnc

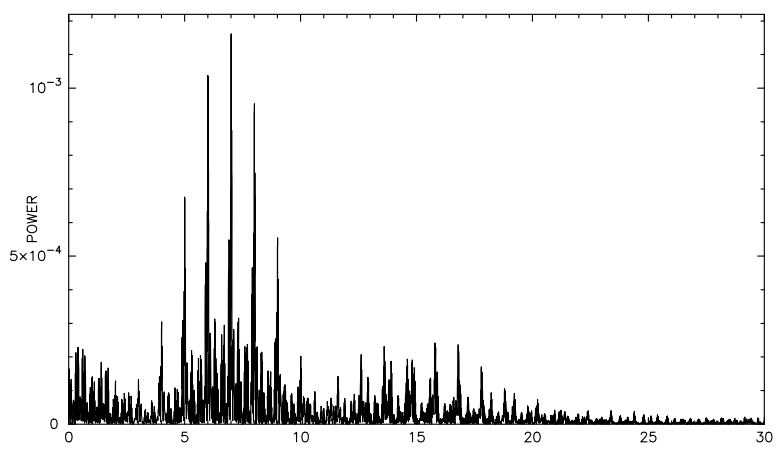

ST(05) $-\mathrm{VZ} \mathrm{Cnc}$

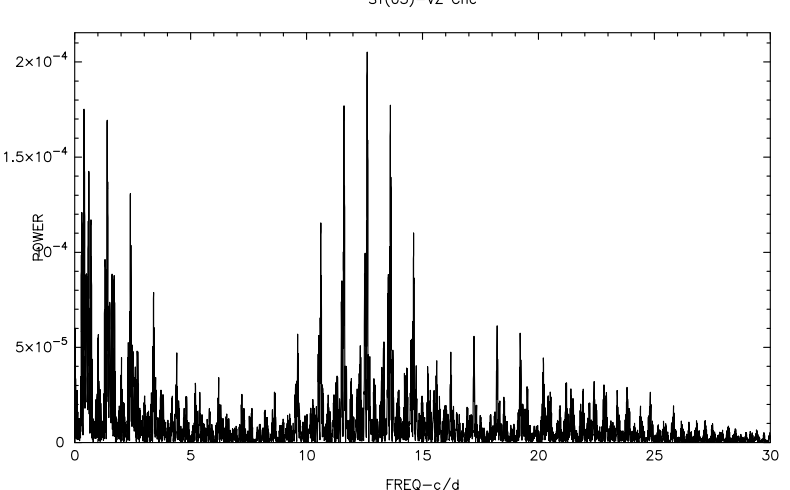

REQ-c/d

Fig. 2. Spectral window and power spectra of the data set VZ1. Multiple pulsation frequencies are extracted one by one 

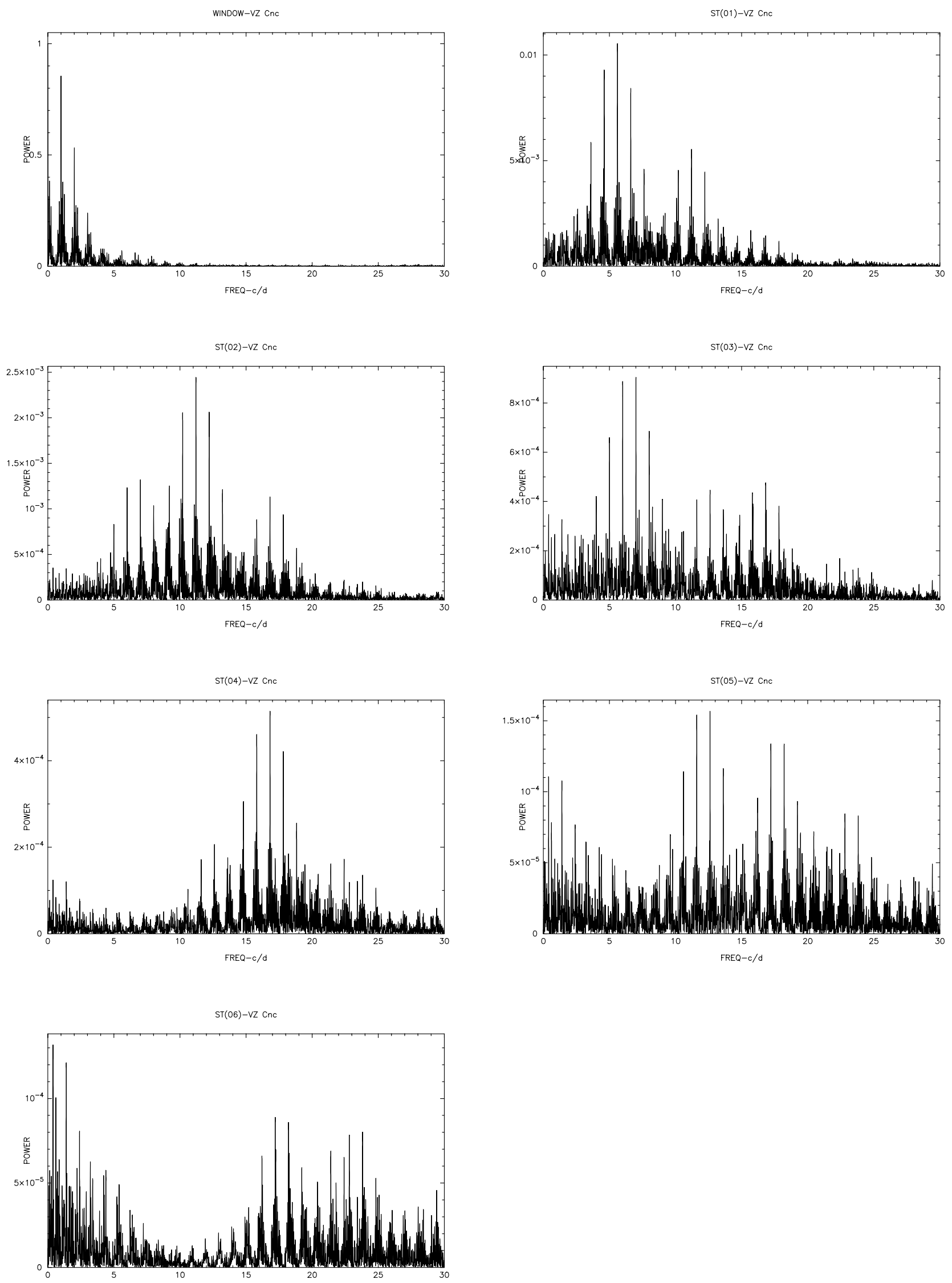

Fig. 3. Spectral window and power spectra of the data set VZ3. Multiple pulsation frequencies are extracted one by one 

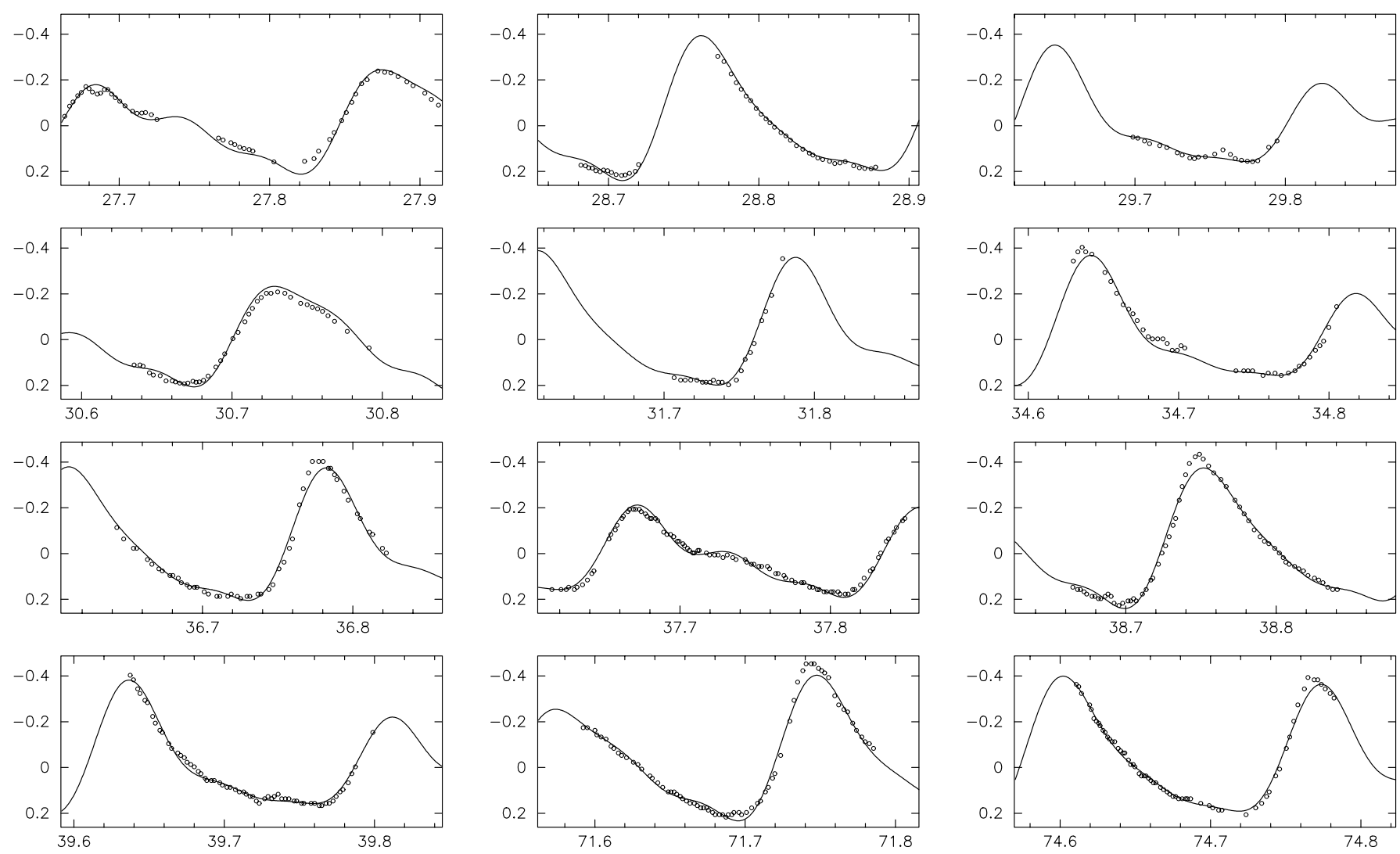

Fig. 4. Observation points and fit curves of VZ1. The abscissa is HJD 2449000.0+, while the ordinate is $\triangle V$ (mag)
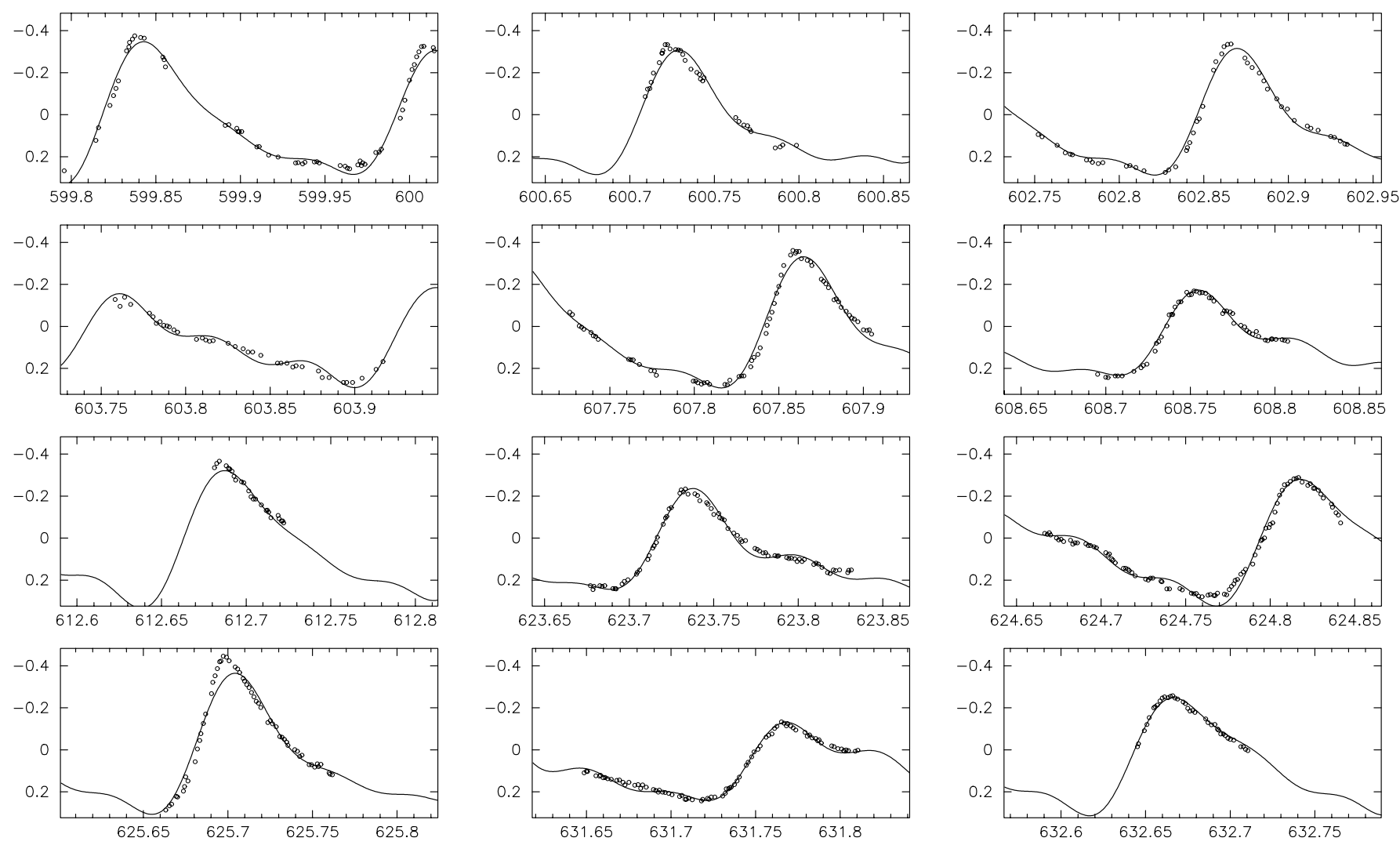

Fig. 5. Observation points and fit curves of VZ3. The abscissa is HJD 2436000.0+, while the ordinate is $\triangle V$ (mag) 

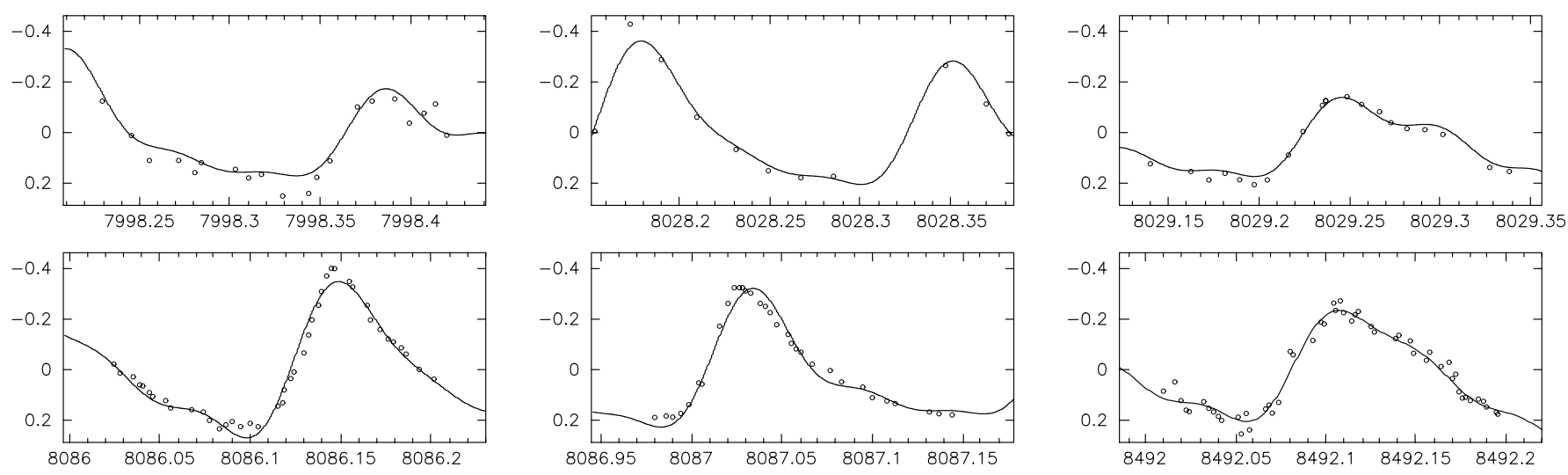

Fig. 6. Observation points and fit curves of VZ2a. The abscissa is HJD 2430000.0+, while the ordinate is $\triangle V$ (mag)
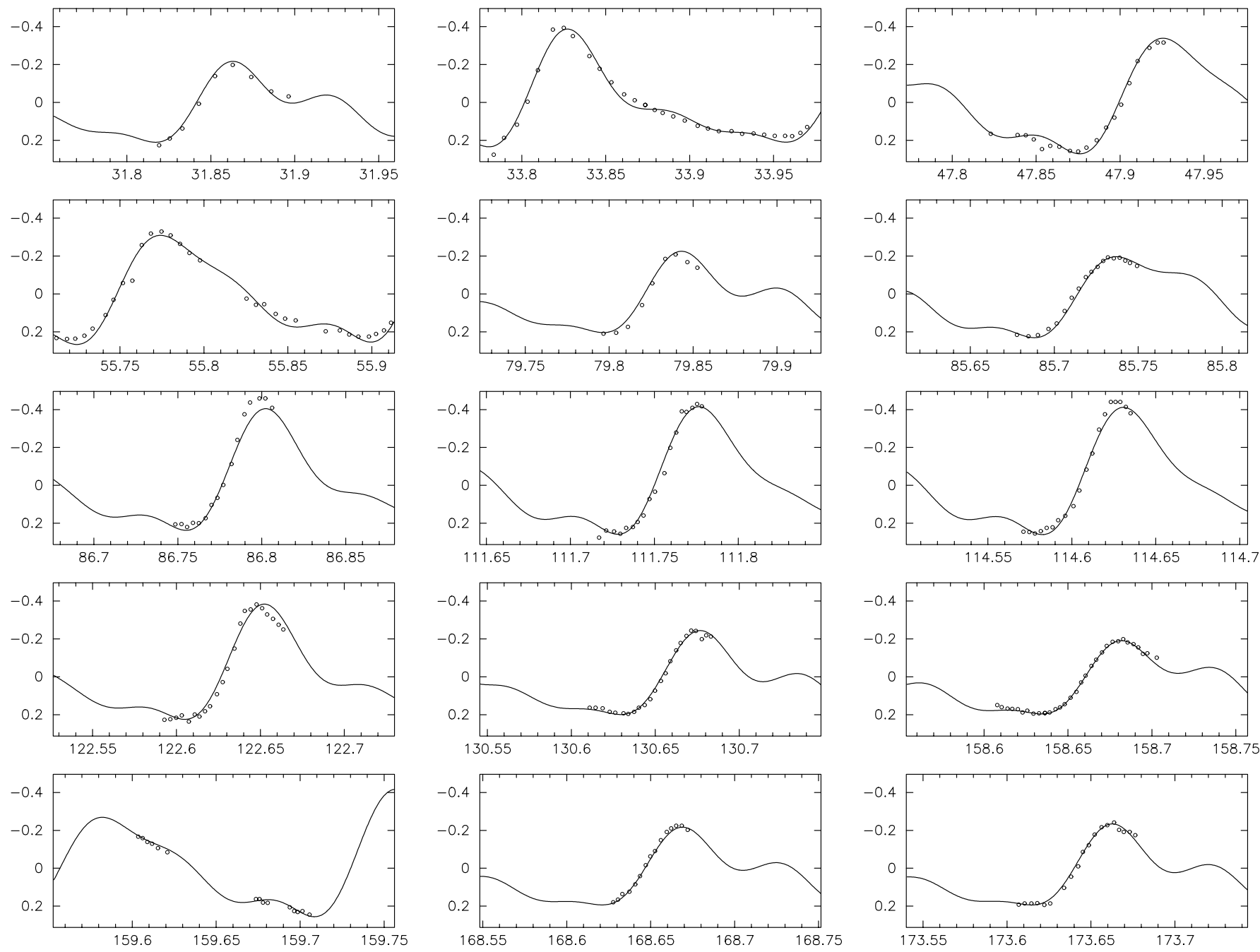

Fig. 7. Observation points and fit curves of VZ4a. The abscissa is HJD 2433600.0+, while the ordinate is $\triangle V$ (mag) 
Table 5. Fitting results for VZ2a, VZ2b, VZ2c and VZ4a, VZ4b, VZ4c with the 6 frequencies

\begin{tabular}{ccccccccc}
\hline No. & Frequency & \multicolumn{6}{c}{ Amplitude (mag) } \\
& (c/d) & VZ 2a & VZ 2b & VZ 2c & VZ 4a & VZ 4b & VZ 4c \\
\hline$f_{1}$ & 5.6066 & 0.1935 & 0.2777 & 0.2497 & 0.2150 & 0.3129 & 0.2051 \\
$f_{2}$ & 11.2128 & 0.0741 & 0.1002 & 0.0907 & 0.0802 & 0.1839 & 0.0915 \\
$f_{3}$ & 7.0022 & 0.0643 & 0.0807 & 0.0838 & 0.0573 & 0.0857 & 0.0750 \\
$f_{4}$ & 16.8188 & 0.0373 & 0.0466 & 0.0494 & 0.0539 & 0.0970 & 0.0529 \\
$f_{5}$ & 12.6095 & 0.0304 & 0.0422 & 0.0431 & 0.0394 & 0.0547 & 0.0456 \\
$f_{6}$ & 1.3957 & 0.0198 & 0.0466 & 0.0374 & 0.0276 & 0.0135 & 0.0311 \\
\hline \multicolumn{2}{l}{ Residual (mag) } & 0.031 & 0.038 & 0.037 & 0.025 & 0.018 & 0.020 \\
\hline
\end{tabular}

(see Table 3) are used to do fitting on data set VZ2a, VZ2b, VZ2c and VZ4a, VZ4b, VZ4c. Table 5 lists the fitting results. Figures 6 and 7 show the observation points and corresponding fitting light curves for VZ2a and VZ4a, respectively. The agreement between the observations and the fitting curves for VZ2b, VZ2c and VZ4b, VZ4c, which are not displayed in this paper, is as good as those for VZ2a and VZ4a, respectively.

\section{Discussion and conclusions}

Studying carefully the frequency values in Table 3, one may find that $f_{1}, f_{3}$ correspond to the periods of 0.17836376 and 0.1428041 days which were identified by Fitch (1955) and Cox et al. (1984), while $f_{6}$ corresponds to the beat period of 0.716292 days. However, more frequencies are proved to be present in the pulsation of VZ Cnc! We may find that $f_{2} \approx 2 f_{1}, f_{4} \approx 3 f_{1}$, and $f_{5} \approx f_{3}+f_{1}, f_{6} \approx f_{3}-f_{1}$. Thus, if we label $f_{1}$ as $\nu_{0}=5.6066 \mathrm{c} / \mathrm{d}$ and $f_{3}$ as $\nu_{1}=7.0022 \mathrm{c} / \mathrm{d}$, the other frequencies can be marked in terms of $\nu_{0}$ and $\nu_{1}$. The identification results are listed in the last column of Tables 3 and 4 within small errors. At the same time, it may be found that the frequencies $\nu_{0}$ and $\nu_{1}$ are usually associated with the first and second overtones of radial pulsation based on the similarity of their period ratio $(0.801)$ to the theoretically expected value of 0.810 . The analysis of the light curves of VZ Cnc also shows that the pulsation frequencies of this star are quite stable while the amplitudes are changing slightly from 1950 to 1993.
The results of multiple frequency analyses show that the star VZ Cnc oscillates simultaneously with two modes of first and second overtones, and some harmonics and cross-frequencies generated by the nonlinear effects. About the large scatter in the $\mathrm{O}-\mathrm{C}$ diagram under the supposition of two modes, although we do not give firm and definitive explanation, the discovery of more than 2 frequencies in the power spectrum of the light curve of this star might give one possibilility.

\section{References}

Ahnert P., 1959, Mitt. uber veranderliche Sterne No. 395

Balona L.A., Stobie R.S., 1983, SAAO Circ. No. 7, 19

Berdnikov L.N., 1975, Perem. Zvezdy 2, 199

Cao M., Jiang S.Y., 1991, Acta Astron. Sin. 32, 401

Cester B., Guiricin B., Mardirossian F., Mezzetti M., Pucillo M., 1977, Inf. Bull. Var. Stars, No. 1338

Cox A.N., McNamara B.J., Ryan W., 1984, ApJ 284, 250

Deeming T.J., 1975, Ap\&SS 36, 137

Ferro A.A., Nuñez N.S., Avila J.J., 1994, PASP 106, 696

Fitch W.S., 1955, ApJ 121, 690

Fitch W.S., Wisniewski W.Z., Johnson H.L., 1966, Comm. Lunar Planet. Lab. 71, 146

Fu J.N., Jiang S.Y., Zhou A.Y., 1998, Publ. Beijing Astron. Obs. 32,35

Guman I., 1955, Budapest Mitteilungen 36, 3

Hahn G., Lustig G., Jasicek H., Klement G., 1975, Inf. Bull. Var. Stars, No. 1078

Hao J.X., 1991, Publ. Beijing Astron. Obs. 18, 35

Jiang S.Y., Yang Z.Z., 1982, Acta Astron. Sin. 23, 333

Joy A.H., Wilson R.E., 1950, PASP 62, 58

López de Coca P., Rolland A., Rodríguez E., Garrido R., 1990, A\&AS 83, 51

Percy J.R., Matthews J.M., Wade J.D., 1980, A\&A 82, 172

Piersimoni A.M., Di Paolantonio A., Burchi R., De Santis R., 1993, A\&AS 101, 195

Ponsen J., Oosterhoff P.T., 1966, Bull. Astron. Inst. Netherlands 18, 459

Popovici C., 1971, Inf. Bull. Var. Stars, No. 508

Spinrad H., 1960, ApJ 131, 134

Todoran I., 1976, IBVS, No. 1141

Whitney B.S., 1950, PASP 62, 56 